\title{
Cytokine Imbalance in a 15-year-old Boy with Pandemic Influenza an Infection Requiring Extracorporeal Membrane Oxygenation
}

\author{
Sang Hyun Park ${ }^{1}$, Chang Min Kang ${ }^{1}$, Dae Seong Kim ${ }^{1}$, Han Ho Kim ${ }^{1}$, Young Yoo ${ }^{1,2}$ \\ 1 Department of Pediatrics, Korea University College of Medicine, Seoul, Korea \\ 2 Allergy Immunology Center, Korea University, Seoul, Korea
}

*Corresponding Author: Dr. Young Yoo, M.D., Ph.D. Department of Pediatrics, Korea University College of Medicine, Goryeodaero 73, Seongbuk-gu, Seoul 02841, Korea, Tel: + 82-2-920-5090, E-mail: yoolina@korea.ac.kr

\begin{abstract}
Acute severe asthma is an acute episode of intractable asthma that is poorly responsive to standard management. Acute respiratory failure or acute respiratory distress syndrome (ARDS) requiring ventilator support may be life-threatening. Although respiratory viral infections are responsible for most asthma exacerbations, the seasonal influenza virus has not been considered a risk factor. However, the 2009 H1N1 influenza A pandemic strain ( $\mathrm{pH} 1 \mathrm{~N} 1)$, which has resulted in numerous hospitalizations and mortalities worldwide, has been reported to be closely related to asthma exacerbation. Thus, with over 300 million people suffering from asthma worldwide, further studies are needed to protect this vulnerable population that is highly susceptible to $\mathrm{pH} 1 \mathrm{~N} 1$ infection. Dysregulation of Th1/Th2 cytokines may be involved in asthma exacerbation. We report the case of a paediatric patient with acute respiratory failure due to asthma exacerbation triggered by pH1 1 infection. The patient exhibited Th2 polarization in the acute phase of exacerbation and recovered from ARDS through timely ventilator support and subsequent extracorporeal membrane oxygenation. Further investigation regarding the mechanism of immune response in asthma exacerbations associated with $\mathrm{pH} 1 \mathrm{~N} 1$ infection may elucidate the optimal treatment for asthma.
\end{abstract}

Keywords: Asthma; Cytokines; Extracorporeal membrane oxygenation; H1N1 Subtype; Influenza A Virus; Respiratory Insufficiency.

\section{INTRODUCTION}

Acute severe asthma is an acute episode of intractable asthma that is poorly responsive to standard therapeutic measures ${ }^{[1]}$. It can rapidly turn into potentially fatal acute respiratory failure and requires time-sensitive recognition and management. Respiratory viral infections are the most frequent triggers of asthma exacerbation in both children and adults ${ }^{[2]}$. Among the various respiratory viruses, rhinovirus, respiratory syncytial virus, and bocavirus are the most frequently reported viruses that may cause asthma exacerbation, but infection with the seasonal influenza virus is not considered a risk factor [3]. However, there have been reports about the relationship between the $2009 \mathrm{H} 1 \mathrm{~N} 1$ influenza A pandemic strain (pH1N1) and exacerbation of asthma ${ }^{[4]}$. Although the immunological mechanisms underlying asthma exacerbation in viral infection have not been fully elucidated, Th1/Th2 cytokine dysregulation has been suggested as the main cause ${ }^{[5,6]}$. We report the case of a 15-year-old boy who recovered from asthma exacerbation triggered by $\mathrm{pH} 1 \mathrm{~N} 1$ infection through veno-venous extracorporeal membrane oxygenation (VV-ECMO). Serum levels of interleukin (IL) 4, IL 5, and interferon gamma were analyzed during hospitalization for Th1/Th2 imbalance.

\section{CASE REPORT}

This case report was approved by the institutional review board of Anam Hospital (IRB No. 2021AN0227). Written informed consent was obtained from the patient and his parents.

A 15-year-old boy arrived at the emergency room with a complaint of persistent dyspnea over 2 days, which started after visiting a karaoke. He had been treated for asthma at our center for 2 years. At the time of the attack, he was on as-needed shortacting beta-agonists (SABAs) without maintenance therapy for a year. Physical examination revealed wheezing and dyspnea, with an oxygen saturation level of $95 \%$ and peak expiratory flow 
rate (PEFR) of $260 \mathrm{~L} / \mathrm{min}$ (55\% of his maximum PEFR). Chest radiography showed mild peri-bronchial infiltration and slightly hyperinflated lungs (Fig. 1A). Nebulized SABA (2.5 mg) and ipratropium bromide $(500 \mu \mathrm{g})$ were administered three times with a gap of 20 min between consecutive administrations, followed by intravenous methylprednisolone (1 mg/kg). Although his PEFR increased to $370 \mathrm{~L} / \mathrm{min}$ (68\%) after the treatment, respiratory symptoms persisted, requiring hospitalization in the general ward for further management.

At $6 \mathrm{~h}$ after hospitalization, the PEFR had reduced to $250 \mathrm{~L} / \mathrm{min}$ (53\%) despite treatment with systemic steroids and as-needed SABA, and the oxygen saturation decreased to $92 \%$. He was administered oxygen via a facial mask at a flow rate of $10 \mathrm{~L} / \mathrm{min}$. His oxygen saturation improved to $98 \%$, but dyspnea worsened. At $12 \mathrm{~h}$ after hospitalization, the PEFR had dropped to $150 \mathrm{~L} / \mathrm{min}$ $(32 \%)$, and he complained of unbearable chest pain and anxiety. He was moved to the intensive care unit for closer observation and further treatment.

Continuous aminophylline was added to intermittent inhaled SABA, but his condition deteriorated with oxygen saturation falling to $78 \%$. Chest radiography showed pneumonic infiltration and atelectasis in the right upper lobe with mild pneumomediastinum and subcutaneous emphysema (Fig. 1B). Arterial blood gas analysis revealed acidosis, hypoxemia, and hypercapnia $(\mathrm{pH}, 7.083$; partial pressure of carbon dioxide $\left[\mathrm{PaCO}_{2}\right], 83.6 \mathrm{mmHg}$; partial pressure of oxygen $\left[\mathrm{PaO}_{2}\right], 81.6$ $\mathrm{mmHg}$; bicarbonate $\left[\mathrm{HCO}_{3}{ }^{-}\right], 25.2 \mathrm{mEq} / \mathrm{L}$; base excess [BE], $-6.3 \mathrm{mEq} / \mathrm{L} ; \mathrm{O}_{2}$ saturation, $89.4 \%$ ). Following the diagnosis of acute respiratory failure due to acute severe asthma, he was placed under mechanical ventilation (continuous mandatory ventilation mode; fraction of inspired oxygen, 1.0; positive endexpiratory pressure, $5 \mathrm{~cm} \mathrm{H}_{2} \mathrm{O}$; respiratory rate, $18 / \mathrm{min}$; tidal volume, $450 \mathrm{~mL}$ ). However, he did not recover from acute respiratory failure $\left(\mathrm{pH}, 7.183 ; \mathrm{PaCO}_{2}, 70.4 \mathrm{mmHg} ; \mathrm{PaO}_{2}, 59.4\right.$ $\mathrm{mmHg} ; \mathrm{HCO}_{3}^{-}, 24.8 \mathrm{mEq} / \mathrm{L} ; \mathrm{BE},-3.2 \mathrm{mEq} / \mathrm{L} ; \mathrm{O}_{2}$ saturation, $89.7 \%)$.

At $24 \mathrm{~h}$ after hospitalization, VV-ECMO was inserted for urgent respiratory support. Hypercapnia resolved immediately, followed by a gradual improvement in hypoxemia (Table 1). Chest computed tomography scan revealed rapidly progressing atelectasis in the right upper and left lower lobes, with multiple pneumonic infiltrations and extensive subcutaneous emphysema (Fig. 1C). Empirical antibiotics (piperacillin/tazobactam plus teicoplanin), intravenous immunoglobulin (1 $\mathrm{g} / \mathrm{kg} / \mathrm{day}$ ), and steroid pulse therapy (1 $\mathrm{g} / \mathrm{kg} / \mathrm{dose}$ ) were started for the management of pneumonia, boosting the immunity, and management of asthma exacerbation, respectively. Real-time polymerase chain reaction test for respiratory viruses was positive for $\mathrm{pH} 1 \mathrm{~N} 1$. Oseltamivir (oral) and peramivir (intravenous) were administered immediately.

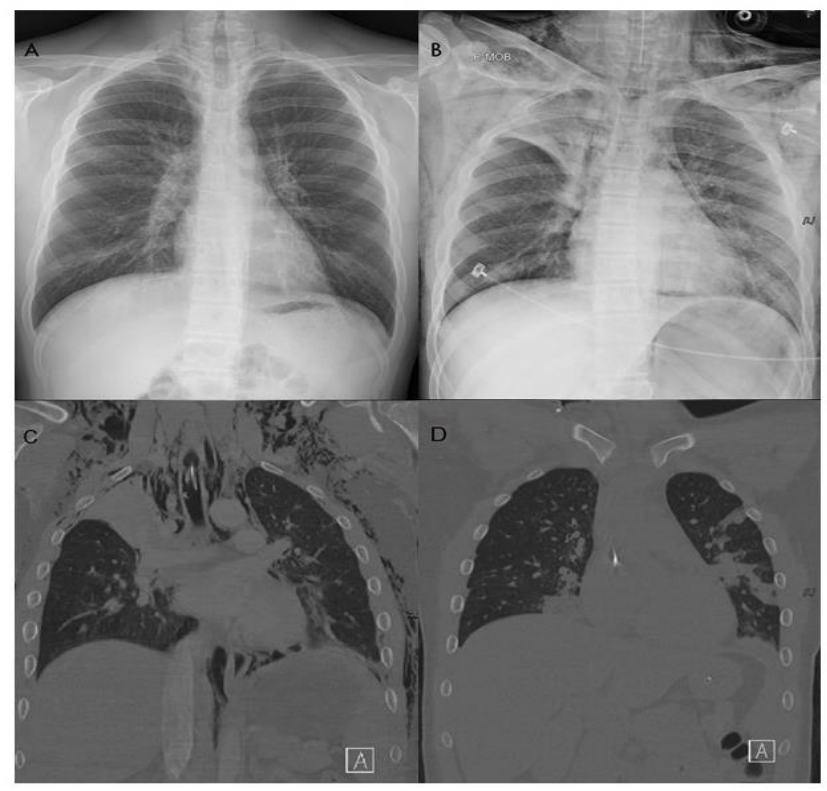

Figure 1: Chest X-rays and CTs with acute and recovery state. (A) Initial CXR at ER shows peri-bronchial infiltration and slightly hyperinflated lungs. Seventeen hours after hospitalization, pneumonia and atelectasis on RUL with mild pneumo-mediastinum and subcutaneous emphysema (C) Twenty-four hours after hospitalization, rapidly progressing atelectasis on $R U L$ and LLL, with multiple pneumonic infiltration and extensive subcutaneous emphysema (D) Hospital day 12 , early recovery state.

Table 1: Timetable of the ABGA results

\begin{tabular}{|l|l|l|l|l|l|l|l|}
\hline $\begin{array}{l}\text { Hospital day } \\
\text { Parameters }\end{array}$ & HD \#1 & $\begin{array}{l}\text { HD \#2 } \\
\text { (ventilated) }\end{array}$ & $\begin{array}{l}\text { HD \#3 (post- } \\
\text { ECMO, 1 hour) }\end{array}$ & $\begin{array}{l}\text { HD \#3 (post- } \\
\text { ECMO, 4 hours) }\end{array}$ & $\begin{array}{l}\text { HD \#3 (post- } \\
\text { ECMO, 7 hours) }\end{array}$ & $\begin{array}{l}\text { HD \#8 (ECMO } \\
\text { weaning) }\end{array}$ & $\begin{array}{l}\text { HD \#27 (ventilator } \\
\text { removed) }\end{array}$ \\
\hline $\mathrm{pH}$ & 7.486 & 7.083 & 7.058 & 7.372 & 7.458 & 7.439 & 7.416 \\
\hline $\mathrm{PaCO}_{2}(\mathrm{mmHg})$ & 26.1 & 83.6 & 116.7 & 39.2 & 36.1 & 38.5 & 31.5 \\
\hline $\mathrm{PaO}_{2}(\mathrm{mmHg})$ & 71.3 & 81.6 & 69.7 & 66.5 & 70.0 & 82.7 & 72.9 \\
\hline $\mathrm{SaO}_{2}(\%)$ & 95.7 & 89.4 & 82.2 & 89.8 & 94.8 & 96.5 & 94.9 \\
\hline
\end{tabular}

Abbreviations: ABGA: arterial blood gas analysis; HD: hospital day; ECMO: extracorporeal membrane oxygenation; PaCO2: partial pressure of arterial carbon dioxide (CO2); $\mathrm{PaO} 2$ : partial pressure of arterial oxygen (O2); SaO2: arterial blood oxygen saturation level.

The patient was on VV-ECMO for 5 days and on mechanical ventilation for 17 days. Chest computed tomography on day 12 showed gradual resolution of atelectasis, pneumonic 
infiltrations, and subcutaneous emphysema (Fig. 1D). The patient recovered slowly but completely after 32 days of admission. During admission, serum cytokine levels were analyzed between day 4 and day 31 of hospitalization for Th1/Th2 cytokine polarization. In the acute phase of asthma exacerbation, a Th2-skewed immune response was relatively predominant, and balance was restored after day 8 of hospitalization (Fig. 2).

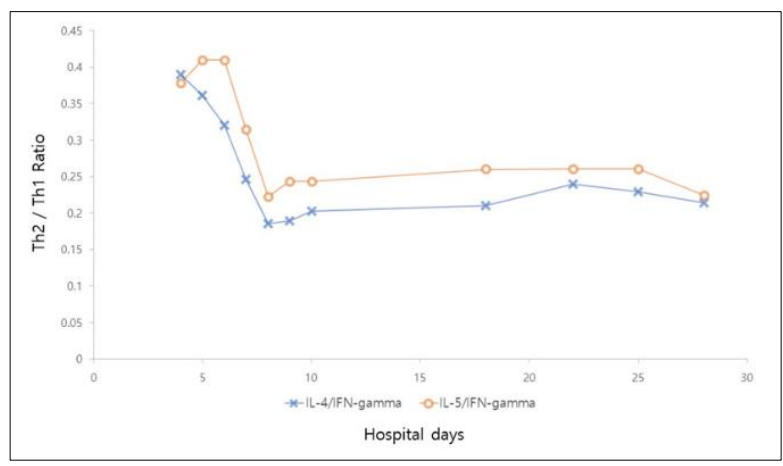

Figure 2: Serum Th2/Th1 cytokine ratio Th2 polarization in the acute phase of asthma exacerbation is prominent from hospital day

After discharge, the patient was symptom-free for 2 years with normal spirometry results. Subsequently, asthma recurred with repeated dyspnea after exercise, requiring daily use of SABA. Spirometry revealed an elevated bronchodilator response (39\%) and positive bronchial hyper-responsiveness on methacholine challenge test $(2.46 \mathrm{mg} / \mathrm{mL}$ of provocative methacholine concentration caused a $20 \%$ reduction in the forced expiratory volume in one second). His symptoms are currently under control with inhaled corticosteroids, long-acting beta-agonists, and leukotriene receptor antagonists as maintenance therapy.

\section{DISCUSSION}

Acute severe asthma, formerly known as status asthmaticus, is a medical emergency characterized by hypoxemia, hypercapnia, and a risk of secondary respiratory failure. In the present case, the patient failed to respond to conventional therapies, despite timely recognition and management. Viral respiratory tract infection is the most common risk factor for asthma exacerbation in both children and adults ${ }^{[3]}$. In the present case, the patient with $\mathrm{pH} 1 \mathrm{~N} 1$ infection showed rapid deterioration requiring emergency VV-ECMO. Timely VV-ECMO saved the patient without any sequelae. However, the mechanisms involved in acute exacerbation remain unclear.

Asthmatic patients are at a higher risk of severe manifestations associated with respiratory viral infections than non-asthmatic individuals ${ }^{[7]}$. Some viral infections cause more severe asthma exacerbation than others, since they trigger different immune reactions, such as a Th2-polarized response, in asthmatic patients compared to those in non-asthmatics individuals [8]. Thus, it is important to verify whether the exacerbation is due to a Th2-polarized response to $\mathrm{pH} 1 \mathrm{~N} 1$ infection. Some studies have focused on host factors, emphasizing the role of a Thskewed response in uncontrolled or exacerbated asthma [6].
Others have pointed out the novel traits of $\mathrm{pH} 1 \mathrm{~N} 1$ including its ability to induce severe pulmonary inflammation or asthmatic symptoms ${ }^{[4,9]}$. Increased susceptibility of asthmatic children to pH1N1 infection has also been observed [10]. Our finding of Th2 predominance in the early phase of exacerbation seems to support the polarized response of an asthmatic host.

In the present case, the patient experienced acute respiratory failure leading to severe acute respiratory distress syndrome (ARDS) according to the Berlin criteria. VV-ECMO is indicated for patients with acute respiratory failure or ARDS who are presumed to have a reversible cause. Timely VV-ECMO may prevent the insult caused by mechanical ventilation, with fewer sequelae. Although many studies have encouraged early use of $E C M O$, the duration of mechanical ventilation before the commencement of ECMO was over 2 days in most studies, and children have been infrequently treated with ECMO [11]. Our patient was treated in a timely manner, enabling full recovery without any sequelae.

In 2009, pH1N1 caused over 12,500 deaths in the United States alone. Asthma was the most common risk factor for severe cases and it was identified in $17 \%-27 \%$ of the hospitalized patients [5]. Attempts to reduce the severity of asthma exacerbation by regulating Th2 cytokines are desirable. Clinical trials using a human monoclonal antibody targeting IL-4 receptor alpha to control asthma have been performed successfully, and the antibody has been found to effectively reduce $\mathrm{pH} 1 \mathrm{~N} 1$-related morbidity in murine models [5]. More extensive studies are needed to derive further conclusions, but the current rise in biologics may provide an additional option to fight against pH1N1 infection and its high respiratory complications, particularly in highly vulnerable populations.

\section{CONCLUSION}

In conclusion, we emphasize the need for early recognition and timely management of $\mathrm{pH} 1 \mathrm{~N} 1$ infection in asthmatic patients. Asthma should be well controlled to avoid potential morbidities. Recent advances in biologics to control asthma might also be beneficial in preventing asthma exacerbations.

\section{Conflict of Interest}

All authors have no potential conflict of interest relevant to this article to report.

\section{Authors' Contribution}

Sang Hyun Park: Conceptualization, Data curation, Formal analysis, Project administration, Visualization, Writing - original draft, Writing - review \& editing,

Chang Min Kang: Data curation, Formal analysis, Project administration,

Dae Seong Kim: Data curation, Formal analysis, Project administration, 
Han Ho Kim: Data curation, Formal analysis, Visualization,

Young Yoo: Conceptualization, Methodology, Visualization, Writing - review \& editing

\section{Acknowledgements}

None. No funding to declare.

\section{REFERENCES}

1. Agnihotri NT, Saltoun C. Acute severe asthma (status asthmaticus). Allergy Asthma Proc 2019; 40:406-9.

2. Papadopoulos NG, Christodoulou I, Rohde G, Agache I, Almqvist $\mathrm{C}$, Bruno $\mathrm{A}$, et al. Viruses and bacteria in acute asthma exacerbations--a GA(2) LEN-DARE systematic review. Allergy 2011; 66:458-68.

3. Fu LS, Tsai MC. Asthma exacerbation in children: a practical review. Pediatr Neonatol 2014; 55:83-91.

4. Shim DH, Park YA, Kim MJ, Hong JY, Baek JY, Kim KW, et al. Pandemic influenza virus, pH1N1, induces asthmatic symptoms via activation of innate lymphoid cells. Pediatr Allergy Immunol 2015; 26:780-8.

5. Shahangian K, Ngan DA, Chen HHR, Oh Y, Tam A, Wen J, et al. IL-4Ralpha blockade reduces influenza-associated morbidity in a murine model of allergic asthma. Respir Res 2021; 22:75.

6. Wardzyńska A, Pawełczyk M, Rywaniak J, Kurowski M, Makowska JS, Kowalski ML. Circulating MicroRNAs and T-Cell Cytokine Expression Are Associated With the Characteristics of Asthma Exacerbation. Allergy Asthma Immunol Res 2020; 12:125-36.

7. Jackson DJ, Johnston SL. The role of viruses in acute exacerbations of asthma. Journal of Allergy and Clinical Immunology 2010; 125:1178-87.

8. Byeon JH, Lee JC, Choi IS, Yoo Y, Park SH, Choung JT. Comparison of cytokine responses in nasopharyngeal aspirates from children with viral lower respiratory tract infections. Acta Paediatr 2009; 98:725-30.

9. Fujimoto $\mathrm{Y}$, Hasegawa $\mathrm{S}$, Matsushige $\mathrm{T}$, Wakiguchi $\mathrm{H}$, Nakamura $\mathrm{T}$, Hasegawa $\mathrm{H}$, et al. Pulmonary inflammation and cytokine dynamics of bronchoalveolar lavage fluid from a mouse model of bronchial asthma during $\mathrm{A}(\mathrm{H} 1 \mathrm{~N} 1)$ pdm09 influenza infection. Sci Rep 2017; 7:9128.

10. Kloepfer KM, Olenec JP, Lee WM, Liu G, Vrtis RF, Roberg KA, et al. Increased $\mathrm{H} 1 \mathrm{~N} 1$ infection rate in children with asthma. Am J Respir Crit Care Med 2012; 185:1275-9.

11. Australia T, Investigators NZEMOI. Extracorporeal Membrane Oxygenation for 2009 Influenza A(H1N1) Acute Respiratory Distress Syndrome. JAMA 2009; 302:1888-95. 